\title{
Miedo al coronavirus, ansiedad y depresión en profesionales de la salud
}

\author{
Ana Lucia Ortega Malla \\ ORCID: https://orcid.org/0000-0003-2521-7321 \\ alortegam13@est.ucacue.edu.ec \\ Universidad Católica de Cuenca \\ Cuenca, Ecuador \\ Susana Janeth Peña Cordero \\ ORCID: https://orcid.org/0000-0002-6526-2437 \\ spena@ucacue.edu.ec \\ Universidad Católica de Cuenca \\ Cuenca, Ecuador
}

\author{
Isabel Cristina Mesa Cano \\ ORCID: https://orcid.org/0000-0003-3263-6145 \\ imesac@ucacue.edu.ec \\ Universidad Católica de Cuenca. \\ Cuenca, Ecuador \\ Andrés Alexis Ramírez Coronel \\ ORCID: https://orcid.org/0000-0002-6996-0443 \\ andres.ramirez@ucacue.edu.ec \\ Universidad Católica de Cuenca. \\ Cuenca, Ecuador
}

Recibido (26/04/21), Aceptado (14/05/21)

\begin{abstract}
Resumen: La pandemia causada por el virus COVID-19 causó sentimientos de miedo, ansiedad y depresión en el personal de salud, expuestos mayormente al riesgo de contraer esta enfermedad. El presente trabajo se centró en determinar el sentimiento de miedo, ansiedad y depresión, experimentado por los profesionales de la salud por el COVID-19. La metodología aplicada se basó en un estudio descriptivocorrelacional, de corte transversal, no experimental. La muestra está constituida por 152 profesionales. Para la recolección de datos se aplicaron los instrumentos para el miedo (FCV-19S), Ansiedad EstadoRasgo (STAI), y Depresión Estado - Rasgo (IDER). Posterior, se realizó una prueba de normalidad K-S y una prueba de correlación Spearman utilizando el programa estadístico Infostat y SPSS 21 para el análisis estadístico. Los resultados revelan que los profesionales de salud presentan niveles de altos de miedo y ansiedad como consecuencia del COVID-19; sin embargo, no presentaron tener niveles altos de depresión.
\end{abstract}

Palabras Clave: COVID-19, miedo, ansiedad, personal de salud.

\section{Fear of coronavirus, Anxiety and Depression in health professionals}

Abstract: The pandemic caused by the COVID-19 virus caused feelings of fear, anxiety and depression in health personnel, who were exposed to the risk of contracting this disease. The present study focused on determining the feelings of fear, anxiety and depression experienced by health professionals due to COVID-19. The methodology applied was based on a descriptive-correlational, cross-sectional, nonexperimental study. The sample consisted of 152 professionals. For data collection, the instruments for fear (FCV-19S), State-Trait Anxiety (STAI), and State-Trait Depression (IDER) were applied. Subsequently, a K-S normality test and a Spearman correlation test were performed using the statistical program Infostat and SPSS 21 for statistical analysis. The results reveal that health professionals present high levels of fear and anxiety as a consequence of COVID-19; however, they did not present high levels of depression.

Keywords:COVID-19, fear, anxiety, health personnel 


\section{Introducción}

El coronavirus 2019 (SARS-CoV-2) conocido generalmente como COVID-19, según se tiene constancia, se registró en la provincia de Hubei, en la ciudad china de Wuhan, y pertenece a la familia de coronavirus del síndrome respiratorio agudo grave (SARS-CoV) y el síndrome respiratorio del Medio Oriente (MERS-CoV), sin embargo, la pandemia de COVID-19 ha causado rangos de diseminación y morbimortalidad mayores a las esperadas [1]. La diseminación de COVID-19 inició desde el continente asiático al europeo, convirtiéndose este en el segundo epicentro de la infección en el primer cuatrimestre del año 2020 [2]. En enero de 2020, la Organización Mundial de la Salud (OMS)[3] declaró al COVID-19 una emergencia de salud pública de interés internacional y el miércoles 11 de marzo del mismo año lo declaró como una pandemia. Para el 28 de octubre de 2020, el COVID-19 se había extendido por todo el mundo, se habían confirmado más de 43 millones de casos y se habían informado más de 1,1 millones de muertes [4],[5]. A partir de esta declaratoria, y con el objetivo de reducir los brotes, los estados globales y locales establecieron una seria de recomendaciones como el lavado de manos, el aislamiento social obligatorio (cuarentena) y el aislamiento de personas afectadas y aquellas consideradas vulnerables. Sin embargo, y a pesar de todas estas recomendaciones la propagación de la COVID-19 parece no cesar, causando daños a nivel psicológico, sanitario, económico, social y político [4], pues hasta la actualidad se han registrado 94,2 millones de casos y más de 2 millones de fallecidos como consecuencia de la COVID - 19 [6].

En Latinoamerica, uno de los países más afectados por la pandemia de la COVID-19, es Ecuador, que registró el primer caso de COVID-19 el 29 de febrero del año 2020 y para el 6 de Julio ya habían 53.424 casos y 8.026 muertes fueron confirmadas, convirtiéndose en el país con mayores registros de muertes en la región [6]. El rápido aumento de casos de COVID-19 involucra también el incremento de preocupación en las comunidades, en su mayoría relacionada con la enfermedad, lo cual juega un papel importante en las reacciones psicológicas y en el comportamiento de las personas que podría ayudar a minimizar el riesgo de desarrollar enfermedades [4]. Además, las noticias sobre el número de muertos, la aceleración de casos y la constante presentación en los medios de comunicación aumenta los temores, frustraciones, impotencia y depresión, ansiedad y miedo en la gente [1] y en el personal médico y sanitario, ya que ellos se enfrentaban en primera línea a la pandemia, atendiendo directamente a los pacientes y teniendo mayor riesgo de contagio [7].

El presente trabajo muestra un análisis del miedo, la ansiedad y la depresión, presentado en los profesionales de la salud por causa de la pandemia de la COVID-19. El estudio se realizó en el Hospital Teófilo Dávila de la provincia de El Oro. Realizar evaluaciones oportunas de la salud mental de los profesionales, puede contribuir con las mejoras de la calidad de la vida del personal de salud, así como mejorar sus actitudes para asegurar una mejor atención al paciente, una mayor confianza en la gestión de sus actividades diarias y mayor tranquilidad en la población en general, ya que un diagnóstico a tiempo, favorece la estabilidad emocional de las personas y el mejor trato con el entorno [8]- [11].

\section{Desarrollo}

Las causas del miedo, ansiedad y depresión de los profesionales de salud están asociadas a las limitaciones de los establecimientos de salud. Como consecuencia de la crisis económica nacional, el Estado redujo el presupuesto en servicios sociales para la población local, generando déficit de medicamentos, insumos, mascarillas, alcohol, gel, entre otros suministros. Por lo tanto, se generó un mayor riesgo de contagio para la población de médicos, enfermeros y demás personal de salud [12]. Además, entre otros problemas que afrontan los trabajadores sanitarios que se enfrentan a la pandemia de la COVID-19 se destacan el alto riesgo de infección, protección inadecuada contra el contagio, exceso de trabajo, frustración, discriminación, aislamientos, problemas emocionales, falta de contacto y agotamiento [1], siendo estos los generadores de un impacto psicológico negativo en los trabajadores sanitarios del área de COVID-19.

Como lo destaca Fofana et al [13], los trabajadores de la salud tienen un alto riesgo de contraer problemas de salud mental a largo plazo seguidos por los niños, los ancianos, y las personas que ya padecen problemas mentales o psicológicos y económicos [13]. Éste riesgo está asociado al enfrentar grandes desafíos como el miedo a infectarse e infectar a otros, las cargas de trabajo pesadas y la falta de equipo de protección personal [14]. La ansiedad y miedo aumentados por la crisis del COVID-19 imponen una carga severa a las capacidades internas de las personas, generando problemas en la toma de decisiones, disrupción y agotamiento nervioso [9]. Aunque la literatura sobre salud mental y COVID-19 es más bien escasa por el momento en el estado ecuatoriano, la literatura existente a nivel mundial indica que la depresión, una mayor ansiedad y el trastorno obsesivo compulsivo han aumentado significativamente debido al COVID-19.

Ante emergencias catastróficas como el COVID-19 y bajo la influencia de diversos factores mentales y objetivos, el personal médico de primera línea, especialmente el personal de enfermería, puede presentar algunos trastornos psicológicos en respuesta al estrés, como ansiedad y depresión [15]. Si bien la información científica sobre la COVID-19 se incrementa constantemente, esta se centra en los aspectos genéticos y epidemiológicos del virus, y en las medidas de salud pública, dejando de lado los posibles efectos en la salud mental. Adicional, con la cobertura de noticias que enfatiza la transmisión rápida y la tasa de mortalidad relativamente alta, el miedo es una respuesta natural.

El miedo puede motivar a las personas a cumplir los mensajes de salud pública que tienen como objetivo reducir la propagación del COVID-19, pero también puede conducir a una serie de consecuencias psicológicas como la ansiedad y psicosociales como los prejuicios [16], generando una vulnerabilidad para el desarrollo de psicopatología, sobre todo en los individuos en riesgo [17]. Además, es de esperar un 
aumento de las preocupaciones y el miedo asociados específicamente a COVID-19 frente a los bloqueos, las recesiones económicas y la gran tensión en la infraestructura básica [18]. Durante la pandemia de COVID-19 donde los países se contagiaron repentinamente y sin ningún tipo de preparación, las personas altamente afectadas, física y mentalmente, son los empleados de salud que siguen a las personas mayores. A esto se le añade la incertidumbre en los protocolos de diagnóstico $\mathrm{y}$ tratamiento, la rápida propagación del virus, las condiciones éticas y de conciencia a la hora de seleccionar al paciente, la carga de trabajo, el no poder ver a los familiares y miedo a contaminar a la familia, los cuales aumentan los niveles de depresión, ansiedad y estrés de los empleados de salud [19]. Por otro lado, el aislamiento mundial, contribuye también a que se desarrolle estrés, trauma postraumático y depresión [20].

\section{Metodología}

La investigación presentada en este trabajo es de carácter cuantitativo, descriptivo, transversal, de campo y no experimental. Incluye además todos los aspectos bibliográficos que dan soporte a los planteamientos presentados.

\section{A.Participantes}

La muestra estuvo compuesta por 152 profesionales de la salud, del área de enfermería, que laboran en el Hospital Teófilo Dávila en la provincia de El Oro, en la ciudad de Machala, en Ecuador. De la muestra total, 89,5\% fueron mujeres $(\mathrm{N}=136)$ y $10,5 \%$ fueron hombres $(\mathrm{N}=16)$. La edad de los participantes oscilaba entre los 24 y 64 años, con una edad media de 40,74 años.

\section{B.Instrumentos}

Se aplicaron los cuestionarios de miedo FCV-19 [21], el cuestionario de Ansiedad Estado-Rasgo (STAI) de Spielberger, Gorsuch y Lushene [22] y el cuestionario de Depresión Estado/Rasgo (IDER) de Spielberger, Buela-Casal, y Agudelo del 2008 [23].

El cuestionario FCV-19S comprende siete ítems, en el que los participantes indicaron su nivel de acuerdo con las afirmaciones utilizando una escala tipo Likert de cinco ítems. Las respuestas incluyeron "totalmente en desacuerdo", "en desacuerdo", "ni de acuerdo ni en desacuerdo", "de acuerdo" y "totalmente de acuerdo". La puntuación mínima posible para cada pregunta es 1 y la máxima es 5 . La puntuación total se calcula sumando la puntuación de cada elemento (que va de 7 a 35$)$.

El cuestionario STAI comprende 20 frases en las que la persona describe cómo se siente en un momento en particular, a través de la escala de Likert de 4 puntos según la intensidad $(0=$ casi nunca/nada; $1=$ algo/a veces; $2=$ bastante/a menudo; $3=$ mucho/casi siempre). Las puntuaciones de ansiedad Estado (A/E) y ansiedad Rango (A/R) se obtienen sumando los ítems correspondientes a las sub variables de ansiedad positivos y negativos tanto para estado (A/E+, A/E-) como para rango $(\mathrm{A} / \mathrm{R}+, \mathrm{A} / \mathrm{R}-)$. Estas puntuaciones pueden variar en un rango de 0 - 60 puntos, donde los valores altos de $\mathrm{A} / \mathrm{E}$ corresponden a un alto grado de ansiedad situacional o transitoria $\mathrm{y}$ valores altos de $\mathrm{A} / \mathrm{R}$ corresponden a una personalidad ansiosa. Se mide los niveles de acuerdo a las puntuaciones 0-19 normal, 20-28 leve, 29-39 alta, y $>40$ severa [22].

El cuestionario IDER presenta 20 ítems, el objetivo de este instrumento es medir el componente afectivo, está basado en el modelo de afectividad positiva y negativa, y para ello incluye las dimensiones de distimia y eutimia. Además, el cuestionario cuenta con las dos sub-escalas de Rasgo y Estado, donde se evalúa de manera diferente la frecuencia y la intensidad de la afectividad negativa y positiva [24]. La puntuación total de cada escala depresión Estado (D/E) y depresión Estado (E/R) se obtiene sumando los resultados de las dos sub-escalas (distimia y eutimia), y varían entre $10 \mathrm{y}$ $40[23]$.

\section{C.Procedimiento}

La evaluación se realizó mediante una encuesta online que contenía, en primera instancia, preguntas para recolectar información sociodemográfica, y posterior los tres instrumentos, los cuales se enfocaban en conocer los rasgos principales de miedo, ansiedad y depresión.

Todo el proceso de recolección de información estuvo soportado por la Universidad Católica de Cuenca, y se llevaron a cabo los procedimientos necesarios para el consentimiento informado, así como el tratamiento ético de la información.

\section{D.Análisis estadístico}

Se realizó un análisis descriptivo de las características sociodemográficas y de miedo al coronavirus, ansiedad y depresión en profesionales de enfermería mediante frecuencias, porcentajes (variables cualitativas) y medidas de tendencia central (variables cuantitativas), se realizó una prueba de normalidad Kolmogorov-Smirnov, para muestra mayor a 50. Se utilizó la prueba Rho de Spearman para realizar el análisis de correlación entre los factores miedo, ansiedad y depresión con variables sociodemográficas. Los análisis estadísticos se ejecutaron mediante el programa estadísticos InfoStat y SPSS21.

\section{Resultados}

\section{A.Descripción de la muestra}

Los resultados descriptivos se presentan en la Tabla 1. En esta se describen las frecuencias y porcentajes de las variables sociodemográficas: genero, edad, residencia, ocupación y estado civil, de la muestra formada por un total de 152 profesionales de enfermería del Hospital Teófilo Dávila. La mayoría de los profesionales de enfermería fueron mujeres $(89,5 \%)$. 
Tabla 1. Datos sociodemográficos de la muestra $\mathrm{N}=152$

\begin{tabular}{|c|c|c|c|}
\hline & & Frecuencia & Porcentaje \\
\hline \multirow{3}{*}{ Género } & Femenino & 136 & 89,5 \\
\hline & Masculino & 16 & 10,5 \\
\hline & Total & 152 & 100,0 \\
\hline \multirow{9}{*}{ Edad } & $<=30$ & 35 & 23,0 \\
\hline & $31-39$ & 49 & 32,2 \\
\hline & $40-47$ & 20 & 13,2 \\
\hline & $48-56$ & 21 & 13,8 \\
\hline & $57-64$ & 27 & 17,8 \\
\hline & Total & 152 & 100,0 \\
\hline & media & 40,74 años & \\
\hline & mínimo & 24 años & \\
\hline & máximo & 64 años & \\
\hline \multirow[t]{3}{*}{ Residencia } & Rural & 22 & 14,4 \\
\hline & Urbana & 130 & 85,6 \\
\hline & Total & 152 & 100,0 \\
\hline \multirow{3}{*}{ Ocupación } & Empleado & 20 & 13,2 \\
\hline & Público & 132 & 86,8 \\
\hline & Total & 152 & 100,0 \\
\hline \multirow{5}{*}{ Estado Civil } & Casado & 68 & 44,7 \\
\hline & Otro & 26 & 17,0 \\
\hline & Soltero & 56 & 36,8 \\
\hline & Viudo & 2 & 1,3 \\
\hline & Total & 152 & 100,0 \\
\hline
\end{tabular}

B.Miedo al COVID-19 de profesionales de enfermería del Hospital Teófilo Dávila

Se efectuó, primero, una prueba de confiabilidad de los instrumentos a partir del alfa de Cronbach. Los datos de confiabilidad corresponden a valores del alfa de Cronbach de 0,$859 ; 0,739$ y 0,505 para las escalas FCV-19S, STAI e IDAR respectivamente. Se consideran estos valores altos y aceptables.

En la Tabla 2 se muestran los resultados obtenidos correspondientes a la media de las puntuaciones de la escala miedo. La media varía desde 19,00 hasta 21,85, caracterizando a la muestra de estudio con alto niveles de miedo. En el estudio se observó que el género masculino presenta un mayor miedo que el femenino, alcanzando un porcentaje de $50 \%$ por encima del $44,1 \%$ en las mujeres; esto puede estar relacionado a las tasas de mortalidad, ya que según datos proporcionados por la Organización Mundial de la Salud (OMS), la tasa de mortalidad por COVID-19 es mayor en hombres que en mujeres, aumentando su preocupación y miedo. Por otro lado, la edad de mayor sensibilidad al miedo fue la comprendida de 31 a 30 años, con un valor porcentual de 51,0\%. En las zonas rurales se observó un 63,6\% mayor miedo que en las zonas urbanas, este resultado se respalda en lo establecido por [25] que indica que las personas que pueden responder con mayor intensidad al estrés de una crisis son aquellas que viven en aislamiento social, incluidas aquellas que viven solas y en áreas rurales o fronterizas. En la evaluación también fue posible observar que los empleados del sector público presentan un nivel de miedo mayor al de los sectores privados, lo cual podría ser un factor importante porque el sector público es más visitado que el privado, tienen mayor aglomeración de personas y menos equipos de seguridad.

Referente al estado civil, con el 50,0\% fueron los solteros los que presentaron mayores niveles de miedo, esto puede estar relacionado a que, en el caso de los padres solteros, durante el brote, estos tienen que hacer frente a muchas decisiones estresantes sin precedentes y hacer malabarismos con las decisiones cotidianas sobre sus hijos y trabajar siguiendo pautas que afectan a la seguridad y la salud de su familia y de otras personas. Por otro lado, la gran mayoría de los progenitores solteros en todo el mundo son mujeres y la carga que soportan las madres trabajadoras nunca es más flagrante que durante las amenazas a la salud pública y las emergencias como la de la COVID-19 [26]. 
Tabla 2. Medias y desviaciones típicas de miedo de la muestra $\mathrm{N}=152$

\begin{tabular}{|c|c|c|c|c|c|}
\hline \multicolumn{6}{|c|}{ Miedo COVID-19 } \\
\hline & & Media & $\begin{array}{c}\text { Desviación } \\
\text { típica }\end{array}$ & $\mathrm{N}$ total & $\begin{array}{c}\% \text { del } \mathrm{N} \text { de la } \\
\text { columna }\end{array}$ \\
\hline \multirow{2}{*}{ Género } & Femenino & 20,89 & 6,29 & 136 & $89,5 \%$ \\
\hline & Masculino & 20,56 & 5,61 & 16 & $10,5 \%$ \\
\hline \multirow{5}{*}{$\begin{array}{c}\text { Edad } \\
\text { (agrupado) }\end{array}$} & $<=30$ & 21,29 & 5,23 & 35 & $23,0 \%$ \\
\hline & $31-39$ & 20,82 & 5,35 & 49 & $32,0 \%$ \\
\hline & $40-47$ & 19,00 & 6,71 & 20 & $13,2 \%$ \\
\hline & $48-56$ & 21,38 & 6,52 & 21 & $13,8 \%$ \\
\hline & $57-64$ & 21,32 & 8,06 & 27 & $17,8 \%$ \\
\hline \multirow{2}{*}{ Residencia } & Rural & 19,73 & 5,06 & 22 & $14,4 \%$ \\
\hline & Urbana & 21,05 & 6,37 & 130 & $85,6 \%$ \\
\hline \multirow{2}{*}{ Ocupación } & Empleado & 20,00 & 8,50 & 20 & $13,2 \%$ \\
\hline & Público & 20,96 & 5,83 & 132 & $86,8 \%$ \\
\hline \multirow{4}{*}{ EstadoCivil } & Casado & 20,81 & 6,46 & 68 & $44,7 \%$ \\
\hline & Otro & 21,85 & 6,63 & 26 & $17,0 \%$ \\
\hline & Soltero & 20,73 & 5,67 & 56 & $36,8 \%$ \\
\hline & Viudo & 13,00 & 1,41 & 2 & $1,3 \%$ \\
\hline
\end{tabular}

C.Ansiedad de profesionales de enfermería del Hospital Teófilo Dávila

Respecto a la ansiedad, en la Tabla 3 se presentan los resultados obtenidos correspondientes a las medias de la escala ansiedad. En cuanto a la ansiedad Estado los mayores porcentajes corresponden a un nivel alto, sin embargo, referente a la ansiedad Rango los mayores porcentajes recaen dentro del nivel normal.

Para cada variable sociodemográfica, los mayores porcentajes de ansiedad corresponden a las mujeres (43,4\%), aquellos entre las edades de 31-39 años (49,0\%), los que residen en el área urbana $(43,8 \%)$, los trabajadores públicos $(45,5 \%)$ y los casados $(45,6 \%)$. Todos estos porcentajes corresponden a un nivel alto de ansiedad. En cuanto a la ansiedad rango, los mayores porcentajes recaen dentro de los considerados niveles normales. Estos resultados sugieren que, si bien la muestra tiene niveles altos de ansiedad, estos son un estado actual más no están predispuestos a desarrollar una tendencia ansiosa. Por lo tanto, los niveles altos de la muestra se pueden reducir con técnicas de relajación [24].

Los resultados obtenidos concuerdan con los resultados de otros trabajos, especialmente en lo referente al género femenino. En el estudio de revisión bibliográfica realizado por Chun-Yi y Lin-Yi [25], cuyo objetivo era identificar las poblaciones vulnerables a la ansiedad y la depresión durante el COVID-19, determinó que el género femenino fue vulnerable a la ansiedad en la mayoría de los estudios. Se encontró además que las personas de 21 a 30 años tenían la mayor ansiedad y niveles de depresión [27].

Resultados semejantes se presentan en [28], en el cual se evaluó el impacto psicológico de la COVID-19 en una muestra de profesionales sanitarios españoles. Dicho estudio puso de manifiesto que los niveles de ansiedad, depresión, estrés e insomnio eran superiores en mujeres, siendo las diferencias estadísticamente significativas para la ansiedad y el estrés. Además, manifiesta que las profesionales sanitarias de mayor edad, revelaron niveles más altos de toda la sintomatología evaluada. El trabajo realizado por Pietri [29] entre marzo y abril del 2020, con una muestra de 660 adultos italianos, encontró que un aumento sustancial de la ansiedad fue mayor en las mujeres como consecuencia de la pandemia del COVID-19. Menciona además que la prensa diaria de las autoridades sanitarias que informan de nuevos casos y defunciones también podrían haber contribuido a un aumento de la ansiedad por la salud y, en consecuencia, de los síntomas de ansiedad.

Por otro lado, Schweda en su estudio [18] que tuvo como objetivo investigar las reacciones psicológicas relacionado con el COVID-19 en una muestra de 15.308 alemanes, determinó que la ansiedad generalizada disminuye con la edad, pero el miedo relacionado con COVID-19 es más pronunciado en participantes de edad avanzada. La ansiedad generalizada también es más frecuente en las comunidades rurales, pero el miedo relacionado con COVID-19 es elevado en las metrópolis.

Adicional, como lo indica [30] el cual basado en una encuesta transversal que incluyó a 1.257 trabajadores de la salud de 34 hospitales de China, reveló alta prevalencia de síntomas de salud mental entre ellos, donde se destaca que $50,4 \%, 44,6 \%, 34 \%$ y 71,5\% de todos los participantes informaron síntomas de: depresión, ansiedad, insomnio y angustia, respectivamente, destaca además que trabajar en la atención médica de primera línea, con participación directa con pacientes con la COVID-19, fue un factor de riesgo independiente para todos los síntomas antes expuestos, por lo que la salud mental de estas personas puede requerir atención 
especial. Similares resultados se obtuvieron en [31].

Tabla 3. Medias y desviaciones típicas de la ansiedad Estado y Rango de la muestra $\mathbf{N}=152$

\begin{tabular}{|c|c|c|c|c|c|c|c|}
\hline & & & & Ans & Estado & Ansieda & Rango \\
\hline & & $\mathrm{N}$ & $\%$ & Media & $\begin{array}{l}\text { Desviación } \\
\text { típica }\end{array}$ & Media & $\begin{array}{c}\text { Desviación } \\
\text { típica }\end{array}$ \\
\hline Género & Femenino & 136 & $89,5 \%$ & 25,81 & 12,24 & 18,85 & 8,83 \\
\hline & $\begin{array}{c}\text { Masculin } \\
\mathrm{o}\end{array}$ & 16 & $10,5 \%$ & 22,81 & 11,85 & 14,56 & 7,42 \\
\hline Edad & $<=30$ & 35 & $23,0 \%$ & 24,63 & 10,23 & 19,29 & 7,76 \\
\hline (agrupado) & $31-39$ & 49 & $32,0 \%$ & 26,82 & 13,94 & 18,78 & 8,47 \\
\hline & $40-47$ & 20 & $13,2 \%$ & 26,20 & 12,58 & 18,15 & 9,27 \\
\hline & $48-56$ & 21 & $13,8 \%$ & 23,71 & 10,87 & 17,43 & 8,56 \\
\hline & $57-64$ & 27 & $17,8 \%$ & 25,11 & 12,39 & 17,54 & 10,61 \\
\hline Residencia & Rural & 22 & $14,4 \%$ & 22,05 & 12,89 & 17,14 & 9,09 \\
\hline & Urbana & 130 & $85,6 \%$ & 26,08 & 12,03 & 18,61 & 8,74 \\
\hline Ocupación & $\begin{array}{c}\text { Emplead } \\
\text { o }\end{array}$ & 20 & $13,2 \%$ & 18,10 & 12,91 & 13,45 & 7,65 \\
\hline & Público & 132 & $86,8 \%$ & 26,58 & 11,77 & 19,04 & 8,66 \\
\hline Es tadoCi vil & Casado & 68 & $44,7 \%$ & 25,45 & 12,50 & 18,16 & 8,79 \\
\hline & Otro & 26 & $17,0 \%$ & 25,04 & 10,89 & 18,12 & 8,72 \\
\hline & Soltero & 56 & $36,8 \%$ & 26,39 & 12,30 & 19,32 & 8,64 \\
\hline & Viudo & 2 & $1,3 \%$ & 8,00 & 5,66 & 4,50 & 2,12 \\
\hline
\end{tabular}

D.Depresión de profesionales de enfermería del Hospital Teófilo Dávila

En cuanto a la depresión, en la Tabla 4 se observa los resultados de las medias de depresión para Estado y Rasgo obtenidos. Las puntuaciones inferiores a al percentil 75 (P75) se consideran normales, mientras las puntuaciones mayores al P75 indican presencia de depresión. Por lo tanto, se obtu- vo que para la depresión estado el P75 es correspondientes a 24 mientras que para la depresión rango el P75 es 25. De acuerdo a los valores presentados, estos reflejan niveles de ansiedad considerados normales para las dos sub-escalas de la depresión: Estado y Rango. Es decir que los profesionales de enfermería del Hospital Teófilo Dávila no crean una susceptibilidad al desarrollo de reacciones depresivas [32].

Tabla 4. Medias y desviaciones típicas de la depresión Estado y Rango de la muestra N=152

\begin{tabular}{|c|c|c|c|c|c|c|c|}
\hline & & \multicolumn{4}{|c|}{ Depresión Estado } & \multicolumn{2}{|c|}{ Depresión Rango } \\
\hline & & $\mathrm{N}$ & $\%$ & Media & $\begin{array}{l}\text { Desviación } \\
\text { típica }\end{array}$ & Media & $\begin{array}{c}\text { Desviación } \\
\text { típica }\end{array}$ \\
\hline \multirow[t]{2}{*}{ Género } & $\begin{array}{c}\text { Femenin } \\
0\end{array}$ & 136 & $89,5 \%$ & 21,74 & 3,33 & 22,95 & 2,76 \\
\hline & $\begin{array}{c}\text { Masculin } \\
0\end{array}$ & 16 & $10,5 \%$ & 20,75 & 2,62 & 21,75 & 2,24 \\
\hline Edad & $<=30$ & 35 & $23,0 \%$ & 21,43 & 3,84 & 22,71 & 2,72 \\
\hline \multirow[t]{4}{*}{ (agrupado) } & $31-39$ & 49 & $32,0 \%$ & 21,45 & 3,22 & 22,90 & 2,67 \\
\hline & $40-47$ & 20 & $13,2 \%$ & 20,45 & 3,46 & 22,25 & 2,57 \\
\hline & $48-56$ & 21 & $13,8 \%$ & 21,86 & 2,46 & 22,52 & 2,48 \\
\hline & $57-64$ & 27 & $17,8 \%$ & 22,89 & 2,75 & 23,46 & 3,17 \\
\hline \multirow[t]{2}{*}{ Residencia } & Rural & 22 & $14,4 \%$ & 23,05 & 3,42 & 24,18 & 3,02 \\
\hline & Urbana & 130 & $85,6 \%$ & 21,40 & 3,20 & 22,60 & 2,62 \\
\hline \multirow[t]{2}{*}{ Ocupación } & $\begin{array}{c}\text { Emplead } \\
0\end{array}$ & 20 & $13,2 \%$ & 22,50 & 2,04 & 23,50 & 1,91 \\
\hline & Público & 132 & $86,8 \%$ & 21,48 & 3,41 & 22,70 & 2,83 \\
\hline \multirow[t]{4}{*}{ EstadoCivil } & Casado & 68 & $44,7 \%$ & 21,86 & 2,99 & 23,28 & 2,67 \\
\hline & Otro & 26 & $17,0 \%$ & 21,77 & 3,98 & 23,08 & 3,31 \\
\hline & Soltero & 56 & $36,8 \%$ & 21,20 & 3,28 & 22,16 & 2,42 \\
\hline & Viudo & 2 & $1,3 \%$ & 24,50 & ,71 & 22,50 & 3,54 \\
\hline
\end{tabular}


Finalmente, basado en el coeficiente de correlación Rho de Spearman, se evidenció una mayor relación entre la ansiedad y miedo, con valore de Rho de Spearman de 0,502 (A/E-miedo) y 0,425 (A/R-miedo). Sin embargo, los niveles de depresión, si bien son significativos muestran una relación negativa. Estos resultados eran de esperarse ya que los niveles de depresión mostrados son normales a diferencia de los niveles altos de miedo y ansiedad.

\section{E.Discusión}

Debido a que los profesionales de enfermería presentan niveles altos niveles de miedo y ansiedad como consecuencia del COVID-19, son necesarias estrategias tempranas para la prevención y el tratamiento de los efectos psicológicos que puede crear una pandemia como la del COVID-19. Lozano [33] presenta algunos consejos para para lograr el bienestar de los profesionales durante y después del COVID-19, de acuerdo a diferentes fases. En la pre-fase, no casos en la unidad, aquí las actualizaciones de la comunicación son clave, además del apoyo a los gerentes que están soportando el estrés. En la fase inicial donde se registra el primer caso y en la fase principal donde se registran muchos casos, es necesario promover el apoyo de los compañeros, rotar a los trabajadores de las funciones de alto estrés a las funciones de menos estrés, unir a los trabajadores sin experiencia con los colegas de alta experiencia y sobre todo realizar sesiones de regulación emocional, además de verificar que haya lo básico como descanso, espacio físico adecuado para la alimentación, dormir bien los días libres [33].

Otra serie de recomendaciones planteadas por [34] están dirigidas a la prioridad del personal de mantener la salud mental, entre ellas se encuentran: mantener o iniciar un programa de actividad física por el beneficio psicológico que este representa; utilizar la respiración profunda ya que respirar profundamente disminuye el estrés y ansiedad; desconectarse del trabajo aplicando técnicas como el mindfulness ya que permite el control del estrés, ansiedad y un mayor bienestar emocional; pedir ayuda profesional y fomentar el contacto con la familia y allegados.

En cuanto a las limitaciones del estudio, cabe destacar que la distribución de la muestra al estar formada por el $89.5 \%$ de mujeres, debe llevarnos a tomar los resultados de diferencias por sexo con cierta cautela. Otra limitación son las pocas variables sociodemográficas consideradas; por lo tanto, y como recomendación para futuros trabajos se sugiere considerar variables como número de hijos, horas laborales, nivel de instrucción y disponibilidad de los equipos de protección personal (EPP). Se sugiere esta última variable, ya que como lo dedujo [35], la ausencia del EPP básico como son las mascarillas N95, mascarillas quirúrgicas, batas descartables, guantes descartables y visores; genera diferentes grados de ansiedad, encontrándose que el $83 \%$ de los profesionales dieron niveles altos de ansiedad.

\section{Conclusiones}

Los trabajadores de enfermería del Hospital Teófilo Dávi- la del cantón Machala, presentan a niveles de altos de miedo y ansiedad como consecuencia del COVID-19; sin embargo, no presentaron tener niveles altos de depresión. Referente a las características sociodemográficas y laborales de los participantes del estudio, se contó con una participación predominante de mujeres por lo que se necesitaría más estudios para ser concluyente en cuanto al impacto del COVID-19 en términos de la variable género.

La pandemia causada por el COVID-19 influye de manera negativa sobre la salud mental de las personas de la población en general, y en particular, sobre los grupos poblacionales más vulnerables como son los trabajadores de salud que están en primera línea. La incertidumbre asociada con esta enfermedad puede agravar la salud mental y consecuentemente afectar al personal de la salud. Esto implica que los trabajadores se deben preparar psicológicamente ante las situaciones adversas que todavía tienen que vivir.

Se considera el presente trabajo como una base para poder crear técnicas y medidas que sean aplicables en los centros de salud u hospitalarios, esto con el objetivo de reducir los altos niveles de miedo y ansiedad a los que están sometidos los trabajadores de enfermería como consecuencia del COVID-19. Por otro lado, sería interesante realizar estudios empíricos sobre la depresión, ansiedad, estrés, miedo, estrés, malestar psicológico y maltrato durante a la emergencia sanitaria por la pandemia de COVID-19 relacionados con aspectos emocionales y realizar estudios de intervención educativa.

\section{Agradecimiento}

A la Coordinadora y Docentes de la Maestría en Gestión del Cuidado de la Universidad Católica de Cuenca y al Laboratorio de Psicometría del Centro de Investigación, Innovación y Transferencia de Tecnología (CIITT).

\section{Fuente de Financiamiento}

Este estudio es autofinanciado

\section{Conflictos de Interés}

No existen conflictos personales, profesionales o de otro tipo.

\section{Referencias}

[1]A. Rodríguez-Quiroga, C. Buiza, y J. Quintero, "Update on COVID-19 and mental health," Rev Med, vol. 13, no. 23, pp.1285-96, 2020.

[2]I. Ramonet. (2020, Abril 25). La pandemia y el sistema mundo [Online]. Disponible: https://mondiplo.com/la-pandemia-y-el-sistema-mundo

[3]Organización Mundial de la Salud. (2020, Abril 17). Q\&A on coronaviruses (COVID-19) [Online]. Disponible: https://www.who.int/emergencies/diseases/novel-coronavirus-2019/question-and-answers-hub/q-a-detail/q-a-coronaviruses

[4]T. Caycho-Rodríguez, J. Ventura-León, y M. Barboza-Palomino, "Design and validation of a scale to measure worry 
for contagion of the COVID-19 (PRE-COVID-19)," Enferm Clin, vol. 19, pp. 1-9, 2020.

[5] University of Hopkins. (2021). Estadísticas de enfermedad por coronavirus [Online]. Disponible: www.universidaddehopkins.com

[6]O. Sarasty, C.E. Carpio, D. Hudson, y P.A. Guerrero-Ochoa, I. Borja, "The demand for a COVID-19 vaccine in Ecuador," Vaccione, vol. 38, no. 5, pp.8090-8, 2020.

[7]El Universo. (2020, Abril 12). Fallecidos en época de coronavirus en Guayaquil [Online]. Disponible: www.eluniverso.com

[8]J. Huarcaya-Victoria, "Consideraciones sobre la salud mental en la pandemia de COVID-19," Rev Peru Med Exp Salud Publica, vol. 37, no. 2, pp.327-34, 2020.

[9]C.R. Mejia, J. Rodriguez-Alarcon, L. Garay-Rios, M. Enriquez-Anco, A. Moreno, K. Huaytan-Rojas, et al, "Percepción de miedo o exageración que transmiten los medios de comunicación en la población peruana durante la pandemia de la COVID-19," Rev Cuba Investig Biomédicas, vol. 39, no. 2, pp. e692, 2020.

[10]L.C. Velázquez, "La COVID-19:reto para la ciencia mundial," An la Acad Ciencias Cuba, vol. 10, no. 2, pp.763, 2020. [11]M.A. Serra, "Infección respiratoria aguda por COVID-19: una amenaza evidente," Rev habanera Cienc Méd, vol. 19, no. 1, pp.1-5, 2020.

[12]Ministerio de Salud Pública. (2021) . Estadística de contagios y defunciones por coronavirus [Online]. Disponible: www.msp.gob.ec

[13]N.K. Fofana, F. Latif, S. Sarfraz, Bilal, M.F. Bashir, y B. Komal, "Fear and agony of the pandemic leading to stress and mental illness: An emerging crisis in the novel coronavirus (COVID-19) outbreak," Psychiatry Res, 2020.

[14]S.X. Zhang, S. Sun, A. Afshar Jahanshahi, A. Alvarez-Risco, V.G. Ibarra, J. Li, et al, "Developing and testing a measure of COVID-19 organizational support of healthcare workers - results from Peru, Ecuador, and Bolivia," Psychiatry Res, 2020

[15]M. Naeim M, A. Rezaeisharif, y S.G. Bagvand, "Strategies to reduce the anxiety and depression of nurses in the special wards of COVID-19," Arch Psychiatr Nurs, vol. 34, no. 6, pp.529-30, 2020.

[16]T. Winter, B.C. Riordan, A.H. Pakpour, M.D. Griffiths, A. Mason, J.W. Poulgrain, et al, "Evaluation of the English Version of the Fear of COVID-19 Scale and Its Relationship with Behavior Change and Political Beliefs," Int J Ment Health Addict, 2020

[17]C. Quiroz, A. Pareja, E. Valencia, Y. Enriquez, J. Leon J, y P. Aguilar, "A novel coronavirus, a novel disease: COVID-19," Horiz Med, vol. 20, no. 2, pp.e1208, 2020.

[18]A. Schweda, B. Weismüller, A. Bäuerle, N. Dörrie, V. Musche, M. Fink, et al, "Phenotyping mental health: Age, community size, and depression differently modulate $\mathrm{CO}$ VID-19-related fear and generalized anxiety," Compr Psychiatry, vol. 104, pp.1-9, 2021.

[19]T.P. Velavan y C.G. Meyer, "The COVID-19 epidemic," Trop Med Int Heal, no. 3, pp.278-80, 2020.
[20]University of Hopkins. (2020). Coronavirus, minuto a minuto: la Universidad Johns Hopkins registra cerca de 150.000 muertes en el planeta [Online]. Disponible: https://www. dw.com/es/coronavirus-minuto-a-minuto-la-universidad-johns-hopkins-registra-cerca-de-150000-muertes-en-el-planeta/a-53156111

[21]D.K. Ahorsu, C.Y. Lin, V. Imani, M. Saffari, M.D. Griffiths, y A.H. Pakpour, "The Fear of COVID-19 Scale: Development and Initial Validation," Int J Ment Health Addict, pp.1-9, 2020.

[22]R.D. Spielberger, R.L Gorsuch, R.E. Lushene, "STAI Cuestionario de ANSIEDAD Estado-Rasgo," IEEE Trans Commun, vol. 23, no. 7, pp.3-14, 2015.

[23]C.D. Spielberger, G. Buela-Casal, D. Agudelo, "Inventario de Depresión Estado/Rasgo (IDER),” TEA Ediciones; 2008.

[24]D.M. Vélez, Y.G. Maquet, y P.L López, "Propiedades Psicométricas del Inventario de Depresión Estado Rasgo (IDER) con una muestra de población general colombiana," Av en Psicol Latinoam, vol. 32, no. 1, pp.71-84, 2014.

[25]R. T. Sataloff, M. M. Johns, y K. M. Kost, "Frente a la pandemia: Garantizar la Seguridad y Salud en el Trabajo," Organización Internacional del Trabajo, 2020.

[26]N. Valero, M. Velez, A. Duran, y M. Torres, “Afrontamiento del COVID-19: estrés, miedo, ansiedad y depresión?", Enfermería Investiga. Investigación, Vinculación, Docencia y Gestión, vol. 5, no. 3, pp.63-70, 2020.

[27]C.Y. Lin, y Y.L Lin, "Anxiety and depression of general population in the early phase of COVID-19 pandemic: A systematic review of cross-sectional studies," Arch Clin Psychiatry, vol. 47, no. 6, pp.199-208, 2020.

[28]M. Santamaria, N. Ozamiz-Etxebarria, I. Redondo, J. Jaureguizar, y M. Picaza, "Impacto psicológico de la COVID-19 en una muestra de profesionales sanitarios españoles," Rev Psiquiatr Salud Ment, vol. 8, no. 20, pp. 3-4, 2020. [29]S. De Pietri y C. Chiorri, "Early impact of COVID-19 quarantine on the perceived change of anxiety symptoms in a non-clinical, non-infected Italian sample," J Affect Disord Reports. 2021

[30]J. Lai, S. Ma, Y. Wang, Z. Cai, J. Hu, N. Wei, et al, "Factors Associated With Mental Health Outcomes Among Health Care Workers Exposed to Coronavirus Disease 2019," JAMA Network Open, vol. 3, no. 3, pp.10, 2020.

[31]J. Hernández Rodríguez, "Impacto de la COVID-19 sobre la salud mental de las personas," Medicentro Electrónica, vol. 24, no. 3, pp.578-94, 2020.

[32]O. Febre Córdova, "Propiedades Psicométricas del Inventario de Depresión Estado - Rasgo en Estudiantes de una Universidad Privada de Piura," Tesis, Facultad de Humanidades, Universidad César Vallejo, Perú, 2018.

[33]A. Lozano, "Impacto de la epidemia del Coronavirus (COVID-19) en la salud mental del personal de salud y en la población general de China," Revista de Neuro-Psiquiatria, vol. 83, no. 1, pp. 51-56, 2020.

[34]M. Bueno, S. Barrientos-Trigo, "Cuidar al que cuida: el impacto emocional de la epidemia de coronavirus en las 
enfermeras y otros profesionales de la salud," Enferm Clin, vol.31, pp.35-9, 2020.

[35]A. Espín-Arguello, "Impacto psicológico por necesida- des de bioseguridad en profesionales de enfermería durante la pandemia covid-19," Dominio de las Ciencias, vol. 5, pp. $11-23,2020$. 